\title{
Relative incremental costs of complications of lobectomy for stage I non-small cell lung cancer
}

\author{
Abraham D. Geller, AB, ${ }^{\mathrm{a}, \mathrm{b}}$ Hui Zheng, PhD, ${ }^{\mathrm{c}}$ Douglas J. Mathisen, MD, ${ }^{\mathrm{b}}$ Cameron D. Wright, MD, ${ }^{\mathrm{b}}$ and \\ Michael Lanuti, $\mathrm{MD}^{\mathrm{b}}$
}

ABSTRACT

Objective: To evaluate the relative incremental cost of complications after lobectomy for stage I non-small cell lung cancer (NSCLC).

Methods: Patients treated with open or video-assisted thoracoscopic surgery (VATS) lobectomy for stage I NSCLC between 2008 and 2014 were selected. A patient registry was queried for all complications recorded during a 90-day postoperative interval. Hospital cost data for each patient was concatenated with clinical data. Linear regression was used to assess the impact on direct hospital costs of specific complications.

Results: Among the 488 patients included in this study, 34\% experienced $\geq 1$ complication and $17 \%$ experienced $\geq 1$ major complication. In patients experiencing complications, atrial arrhythmia (13\%), prolonged air leak $(8.6 \%)$, atelectasis $(6.4 \%)$, and transfusion requirement $(4.5 \%)$ were most common. Minor complications increased the relative cost of lobectomy by $29 \%$ (95\% confidence interval $[\mathrm{CI}], 23 \%-34 \% ; P<.001)$ compared to the cost of an uncomplicated lobectomy. Major complications increased costs by $57 \%(95 \% \mathrm{CI}, 53 \%-62 \% ; P<.001)$. The greatest predictor of increased 90-day cost was major pulmonary complications, which increased cost by $111 \%$ (95\% CI, 96\%-126\%; $P<.001)$. Prolonged air leak increased relative mean cost by $22 \%(95 \%$ CI, $10 \%-33 \% ; P<.001)$ and pneumonia by $96 \%$ (95\% CI, 75\%-117\%; $P<.001)$.

Conclusions: Complications, both major and minor, contribute significantly to the total 90-day direct hospital cost of lobectomy for stage I NSCLC. Analysis of 90-day postoperative outcomes more accurately captures costs. Major pulmonary complications, atrial arrhythmia, pneumonia, and prolonged air leak represent 4 high-yield targets for cost reduction. Efforts to control health care spending while improving patient outcomes might optimally focus on reducing complications that incur the greatest relative incremental cost. (J Thorac Cardio-

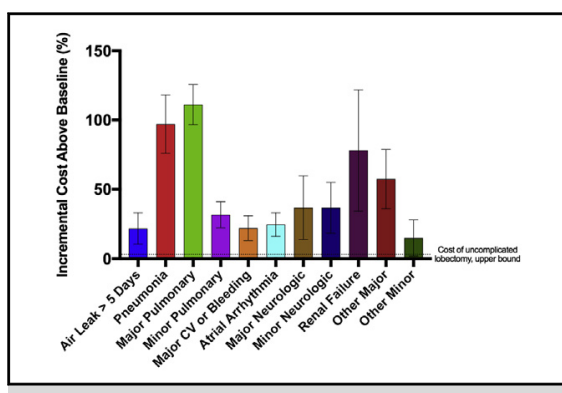

Incremental costs of specific complications after lobectomy for NSCLC.

\section{Central Message}

Preventable complications increase the 90-day cost of lobectomy in varying but significant ways. Reducing the incidence of high-impact complications may decrease costs while improving patient outcomes.

\section{Perspective}

With health care costs growing at an unsustainable rate and increasing scrutiny of outcomes as a measure of performance, there is growing need to understand the cost impact of postoperative complications. This study aims to describe the relative incremental cost of complications of lobectomy for stage I non-small cell lung cancer and relate cost reduction to patient outcome improvement.

See Editorial Commentary page 1812. vasc Surg 2018;155:1804-11)

From the ${ }^{\mathrm{a} H a r v a r d}$ Medical School, Boston, Mass; and ${ }^{\mathrm{b}}$ Division of Thoracic Surgery, and ${ }^{c}$ Biostatistics Center, Massachusetts General Hospital, Boston, Mass.

The statistical analysis in this study was funded by Harvard Catalyst, the Harvard Clinical and Translational Science Center, the National Center for Research Resources, National Center for Advancing Translational Sciences, and the National Institutes of Health (Grant 8UL1TR000170-05), as well as financial contributions from Harvard University and its affiliated academic health care centers.

Read at the 43rd Annual Meeting of The Western Thoracic Surgical Association, Colorado Springs, Colorado, June 21-24, 2017.

Received for publication July 12, 2017; revisions received Oct 19, 2017; accepted for publication Nov 11, 2017; available ahead of print Dec 16, 2017.

Address for reprints: Michael Lanuti, MD, 55 Fruit St, Founders 7, Boston, MA 02114 (E-mail: mlanuti@mgh.harvard.edu).

$0022-5223 / \$ 36.00$

Copyright (c) 2017 by The American Association for Thoracic Surgery

https://doi.org/10.1016/j.jtcvs.2017.11.025
Recently, there has been growing interest in the cost impact of complications following surgery, fueled in part by the rapid increase in national health care spending and efforts to curb excess costs while improving patient outcomes. A recent study estimated that health care spending is

- Scanning this $\mathrm{QR}$ code will take you to a supplemental video for this article. 


\section{Abbreviations and Acronyms \\ CI = confidence interval \\ ERP = enhanced recovery pathway \\ $\mathrm{IQR}=$ interquartile range \\ LOS = length of stay \\ NSCLC $=$ non-small cell lung cancer \\ VATS = video-assisted thoracoscopic surgery}

increasing 2.5 times more rapidly than the US gross domestic product, and that nearly 1 in 3 health care dollars is spent on surgery and related care. ${ }^{1}$ Although national efforts have long sought to contain health care spending, ${ }^{2}$ studies have only recently begun to shed light on the specific cost impact of various surgical procedures and their complications. These studies have demonstrated that complications following lobectomy increase total costs in predictable ways. $^{3-6}$ However, to date, there is a lack of data quantifying the cost impact of specific complications, both major and minor, over a 90-day postoperative interval.

The purpose of this study was to quantify the incidence of several commonly encountered complications following the most common operation for lung cancer (lobectomy), and to estimate the relative incremental direct hospital cost of relevant specific complications. By considering both major and minor complications in patients undergoing both thoracotomy and video-assisted thoracic surgery (VATS), and by incorporating a 90-day postoperative window, we improved our understanding of the detailed cost implications of surgical complications after lobectomy. Specifically, this data may direct quality improvement efforts to optimize patient outcomes and target specific complications for cost reduction (see Video 1 for more details).

\section{METHODS \\ Data Collection}

This was a retrospective cohort study of patients undergoing routine lobectomy for stage I non-small cell lung cancer (NSCLC) at a single institution between 2008 and 2014. All clinical data were extracted from hospital records compiled for inclusion in a national surgical patient database. The records include demographic information as well as diagnoses, procedures, length of stay (LOS), comorbidities, and intraoperative and postoperative complications. To correlate complications with cost, financial records were independently queried from the hospital's billing department, then concatenated with the corresponding clinical data per patient medical record number. Direct hospital costs incurred during a 90-day window (beginning on the date of surgery) were included in the query. This period included the encounter in which the lobectomy took place, as well as subsequent encounters within the first 90 days postoperatively. Total direct hospital cost was reported on a per-encounter level and summarized to a total 90-day direct cost for each patient. Approval for this study was obtained from the Partners Human Research Committee Institutional Review Board (IRB \#2015P002021, activated October 13, 2015), which waived the need for informed consent.

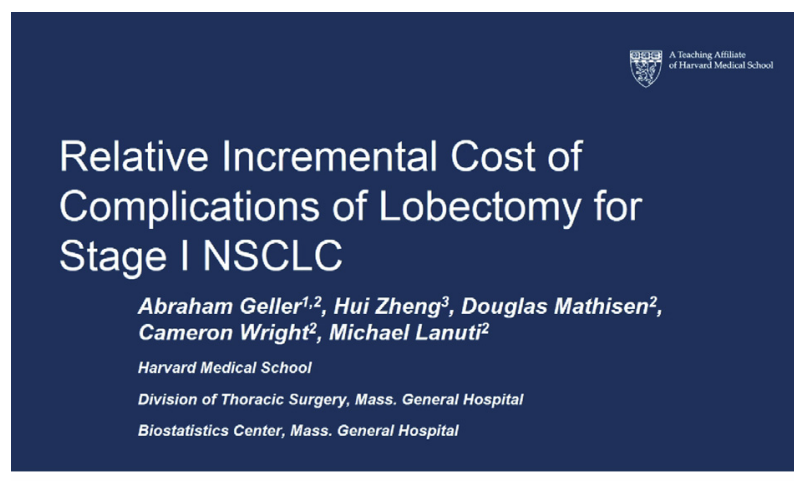

MC̈H MASSACHUSETTS THORACIC SURGERY

VIDEO 1. Presentation of manuscript findings at 43rd annual Western Thoracic Surgical Association annual meeting; slides with audio recording. Video available at: http://www.jtcvsonline.org/article/S0022-5223(17) 32702-2/fulltext.

\section{Patient Selection}

Patients undergoing open or VATS lobectomy for NSCLC between 2008 and 2014 (inclusive) were selected for inclusion in this study. No patients underwent robotic lobectomy at our institution during the study period. Patients were identified from a prospectively collected institutional database of lobectomy cases. Those undergoing lobectomy for any indication other than NSCLC were excluded. Lobectomies performed before January 1, 2008, were also excluded due to technical limitations of the financial dataset. Patients were excluded if their cancer was advanced beyond stage I by clinical staging to minimize the potential confounding effects on adverse outcomes-and cost - of adjuvant chemotherapy or radiation. The query was restricted to selected patients from only a single institution rather than from all institutions pooled in the database, to maintain consistency of complication-cost relationships. As a quality control, patients were further excluded if 90-day follow up data, either clinical or financial, were unavailable or incomplete.

\section{Outcomes and Analysis}

Outcomes measured in this study included the frequency of specific complications, the relative incremental cost of major versus minor complications (ie, the above-baseline attributable cost), and the relative incremental cost of specific complications and complication subtypes, classified as either major or minor. In the analysis of cost, hospital billing department records were queried for total direct hospital costs, which were summarized for all encounters occurring between the day of surgery and postoperative day 90 (inclusive). This includes both fixed direct costs and variable direct costs. Indirect costs were not included in the analysis.

Complication frequency was assessed by dividing the cohort incidence of each complication by the total number of patients in the cohort. Relative incremental cost was defined as cost divided by the cost of an uncomplicated lobectomy, expressed as a percentage, such that a cost of $100 \%$ represents the mean total 90-day direct hospital cost of an uncomplicated lobectomy. The cost of uncomplicated lobectomy refers to the mean total direct hospital cost of the encounter containing the lobectomy without complications. Included in this figure is some cost contribution from hospital stay, medications, and so on, which are necessary components of the hospital encounter for even an uncomplicated lobectomy. Absolute dollars were not reported in this analysis due to the regional variation of health care delivery across the United States that renders the data less translatable. 
TABLE 1. Patient characteristics stratified by surgical approach

\begin{tabular}{|c|c|c|c|}
\hline Characteristic & $\begin{array}{c}\text { Open } \\
(\mathbf{n}=179)\end{array}$ & $\begin{array}{c}\text { VATS } \\
(\mathbf{n}=\mathbf{2 5 8})\end{array}$ & $\begin{array}{c}P \\
\text { value }\end{array}$ \\
\hline Age, $y$, mean \pm SD & $66.5 \pm 12.2$ & $68.2 \pm 10$ & .137 \\
\hline Female sex, n $(\%)$ & $106(59.9)$ & $160(62.5)$ & .650 \\
\hline \multicolumn{4}{|l|}{ Race, n (\%) } \\
\hline White & $163(92.1)$ & $241(94.1)$ & .156 \\
\hline Asian & $9(5.1)$ & $7(2.7)$ & \\
\hline Black & $1(0.6)$ & $5(2)$ & \\
\hline Hispanic or other & $7(4)$ & $5(2)$ & \\
\hline \multicolumn{4}{|l|}{ Smoking status, n (\%) } \\
\hline Never & $28(15.8)$ & 45 (17.6) & .936 \\
\hline Former & $118(66.7)$ & $168(65.6)$ & \\
\hline Current & $31(17.5)$ & $43(16.8)$ & \\
\hline Pack-years, mean $\pm S D$ & $46.37 \pm 31$ & $43.41 \pm 26.4$ & .360 \\
\hline Performance status, n (\%) & & & $<.001 *$ \\
\hline 0 & $79(44.6)$ & $159(62.1)$ & \\
\hline 1 & $97(54.8)$ & 97 (37.9) & \\
\hline 2 & $1(0.6)$ & $0(0.0)$ & \\
\hline \multicolumn{4}{|l|}{ Comorbidity, n (\%) } \\
\hline Hypertension & $98(55.4)$ & 137 (53.5) & 648 \\
\hline Congestive heart failure & $0(0.0)$ & $0(0.0)$ & N/A \\
\hline Coronary artery disease & $15(8.5)$ & $38(14.8)$ & .108 \\
\hline Diabetes mellitus & $18(10.2)$ & 35 (13.7) & .443 \\
\hline Peripheral vascular disease & $14(7.9)$ & $15(5.9)$ & .258 \\
\hline Cerebrovascular disease & $10(5.6)$ & $13(5.1)$ & .964 \\
\hline Steroid use & $1(0.6)$ & $3(1.2)$ & .614 \\
\hline Median length of stay, $d$ & 6.0 & 4.0 & $<.001$ \\
\hline
\end{tabular}

VATS, Video-assisted thoracoscopic surgery; $S D$, standard deviation; N/A, not applicable. *Significant at $\alpha=0.05$

To determine the impact of major versus minor complications on cost, linear regression was used to model the dependence of cost on major and minor complications. Results are reported as means with $95 \%$ confidence intervals (CI). All figures display means, with error bars representing $95 \%$ CIs. Univariate linear modeling was used to assess the additive impact of increasing complications on cost, using Sidak correction for multiple comparisons and controlling for surgical approach. Risk factors for postoperative complications were assessed using multivariate logistic regression with the dichotomous outcomes no complications and $\geq 1$ complication.

In the secondary analysis, complications were subdivided further per primary organ system(s) involved, and multivariable linear regression was used to model the impact of each category on total 90-day direct hospital cost.

\section{RESULTS}

This retrospective cohort analysis spanned 2008 to 2014 and included 488 patients undergoing lobectomy for stage I NSCLC. Patient characteristics are summarized in Table 1. A total of 305 complications were seen in these patients, the distribution of which is summarized in Table 2. Of the 488 patients included in the analysis, 169 patients (34\%) experienced at least 1 complication, and 82 patients $(17 \%)$ experienced at least 1 major complication. The most common complications were atrial arrhythmia, prolonged air leak, and minor pulmonary complications (including pneumothorax, atelectasis requiring bronchoscopy, pleural effusion, and other pulmonary events not otherwise specified). Atrial arrhythmia occurred in 64 patients $(13.1 \%)$ and represented $21 \%$ of all complications in the cohort. Prolonged air leak occurred in 42 patients $(8.6 \%)$, accounting for $13.8 \%$ of all complications. Minor pulmonary complications, which occurred in 60 patients $(11.3 \%)$, accounted for $19.7 \%$ of all complications observed.

The relative cost of major versus minor complications was then modeled by linear regression. Major complications on average increased the cost of lobectomy by $57.4 \%(95 \%$ CI, $52.7 \%-62 \% ; P<.001)$ per complication, whereas minor complications increased the cost of lobectomy by an average of $28.9 \%$ (95\% CI, $23.5 \%$ $34.3 \% ; P<.001$ ) per complication (Figure 1 ). The additive impact of increasing complications on total cost is summarized in Figure 2. No difference in additive cost impact was found between patients who underwent lobectomy via an open thoracotomy approach and those who did so via a VATS approach (Figure 3), except at the level of zero complications, where the VATS approach was still associated with a decreased median LOS compared with thoracotomy (4 days [interquartile range (IQR), 3-5] versus 5 days [IQR, 4-6]; $P=.002$ ). Patients undergoing VATS lobectomy were less likely to experience complications than those undergoing open thoracotomy, even after controlling for other risk factors (odds ratio, $0.48 ; 95 \%$ CI, 0.30-0.77; $P=.002$ ).

Secondary analysis examined the relative incremental cost of various subgroups of complications. The cost of complications was modeled using multivariable linear regression (Figure 4). The complication associated with the greatest relative incremental cost was major pulmonary complications (excluding pneumonia and prolonged air leak), which increased total cost by $111 \%$ above baseline (95\% CI, 97\%-126\%; $P<.001)$, yet occurred in only $2.9 \%$ of patients and accounted for only $4.6 \%$ of all complications observed. Atrial arrhythmia, the most common complication, affecting $13.1 \%$ of patients, increased cost by $25 \%$ (95\% CI, $16 \%-33 \% ; P<.001)$. Of interest, the mean LOS was 4.6 days for patients with uncomplicated lobectomy compared with 5.6 days for patients with postoperative atrial arrhythmia (and no other complications $)(P=.001)$. Prolonged air leak, which occurred in $8.6 \%$ of patients and accounted for $13.8 \%$ of all complications, increased 90-day cost by $21.8 \%(95 \%$ CI, $10.5 \%-33.1 \% ; P<.001)$. Other minor pulmonary complications, which affected $11.3 \%$ of patients and accounted for $19.7 \%$ of all complications, increased cost by $31.7 \%$ (95\% CI, $22.4 \%-41.1 \% ; P<.001)$. Pneumonia occurred in only $2.7 \%$ of patients, accounting for only $4.3 \%$ of all complications, yet the relative incremental 
TABLE 2. Complication list with per event frequency

\begin{tabular}{|c|c|c|c|}
\hline Complication & Incidence, $\mathbf{n}$ & Patients affected, $\%(n=488)$ & Proportion of all complications, $\%(n=305)$ \\
\hline Major pulmonary complications* & 63 & 11 & 20.7 \\
\hline Prolonged air leak $(>5 \mathrm{~d})$ & 42 & 8.6 & 13.8 \\
\hline Pneumonia & 13 & 2.7 & 4.3 \\
\hline Complicated pleural effusion & 2 & 0.4 & 0.7 \\
\hline Acute respiratory distress syndrome & 1 & 0.2 & 0.3 \\
\hline Bronchopleural fistula & 0 & 0 & 0 \\
\hline Pulmonary embolus & 1 & 0.2 & 0.3 \\
\hline Tracheostomy & 4 & 0.8 & 1.3 \\
\hline Respiratory failure & 3 & 0.6 & 1 \\
\hline Re-intubation & 3 & 0.6 & 1 \\
\hline Minor pulmonary complications & 60 & 11.3 & 19.7 \\
\hline Atelectasis & 31 & 6.4 & 10.2 \\
\hline Pneumothorax & 8 & 1.6 & 2.6 \\
\hline Continued ventilator requirement & 1 & 0.2 & 0.3 \\
\hline Other pulmonary events not otherwise specified & 20 & 4.1 & 6.6 \\
\hline Major cardiac or bleeding complications & 33 & 5.1 & 10.8 \\
\hline Myocardial infarction & 1 & 0.2 & 0.3 \\
\hline Ventricular arrhythmia & 1 & 0.2 & 0.3 \\
\hline Deep venous thrombus & 2 & 0.4 & 0.7 \\
\hline Transfusion requirement & 32 & 4.5 & 10.5 \\
\hline Minor cardiac complications & 65 & 13.3 & 21.3 \\
\hline Atrial arrhythmia & 64 & 13.1 & 21 \\
\hline Pericarditis & 1 & 0.2 & 0.3 \\
\hline Major neurologic complications & 8 & 1.6 & 2.6 \\
\hline Central nervous system event & 2 & 0.4 & 0.7 \\
\hline Recurrent laryngeal nerve paralysis & 6 & 1.2 & 2 \\
\hline Delirium & 13 & 2.7 & 4.3 \\
\hline Major infection & 4 & 0.8 & 1.3 \\
\hline Sepsis & 2 & 0.4 & 0.7 \\
\hline Empyema & 2 & 0.4 & 0.7 \\
\hline Minor infection & 20 & 4.1 & 6.6 \\
\hline Urinary tract infection & 19 & 3.9 & 6.3 \\
\hline Wound infection & 1 & 0.2 & 0.3 \\
\hline Infection not otherwise specified & 0 & 0 & 0 \\
\hline New renal failure & 3 & 0.6 & 1.0 \\
\hline Other major complications & 10 & 2 & 3.3 \\
\hline Chylothorax & 2 & 0.4 & 0.7 \\
\hline Other event requiring surgery & 8 & 1.6 & 2.6 \\
\hline Other minor complications & 22 & 4.3 & 7.2 \\
\hline Postoperative ileus & 2 & 0.4 & 0.7 \\
\hline Other gastrointestinal event & 4 & 0.8 & 1.3 \\
\hline Urinary retention & 12 & 2.4 & 4 \\
\hline Other event not requiring surgery & 3 & 0.6 & 1 \\
\hline
\end{tabular}

*Significant at $\alpha=0.05$.

cost of pneumonia was $97.1 \%$ (95\% CI, $76 \%-118 \%$; $P<.001)$. Other statistically significant complications and their associated relative incremental costs were renal failure (defined as both creatinine $>2$ and creatinine $>2$ times baseline) at $78.1 \%(95 \%$ CI, 34.4\%-121.8\%; $P<.001$ ), major cardiovascular and bleeding events at $22.1 \%(95 \%$ CI, $13.2 \%-31.0 \% ; P<.001)$, major neurologic events at $36.8 \%$ (95\% CI, $13.9 \%-59.6 \%$; $P=.002)$, minor neurologic events at $36.8 \%(95 \% \mathrm{CI}$, $18.6 \%-55 \% ; P<.001)$, other major events at $57.4 \%$ (95\% CI, 36\%-78.8\%; $P<.001$ ), and other minor events at $15 \%(95 \%$ CI, $1.9 \%-28.1 \% ; P=.025)$. Major infections and minor infections did not meet statistical significance. 


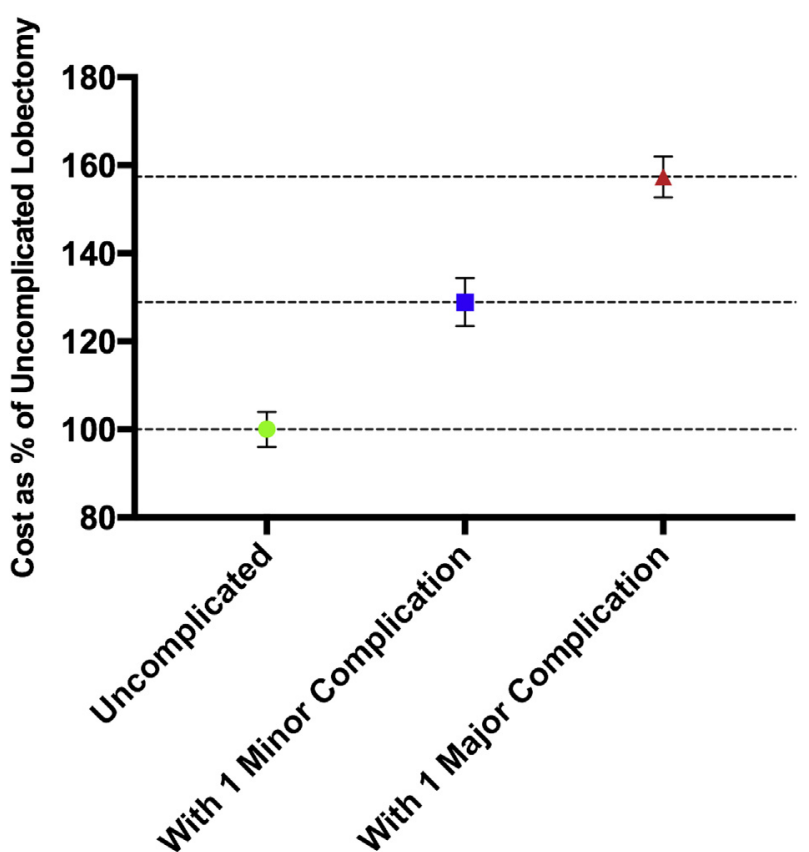

FIGURE 1. Per-event cost impact of major versus minor complications. Data points reflect means with $95 \%$ confidence intervals (CIs). By definition, uncomplicated lobectomy had a relative cost of $100 \%(95 \%$ CI, 96.1\%-103.9\%; $P<.001)$. Incurring 1 minor complication increased the relative cost by $28.9 \%(95 \%$ CI, $23.5 \%-34.3 \% ; P<.001)$ and incurring one major complication increased relative cost by $57.4 \%$ $(95 \%$ CI, 52.7\%-62.0\%; $P<.001)$ above baseline.

\section{DISCUSSION}

In this retrospective cohort study, we have identified the relative incremental cost impact of complications of lobectomy for stage I NSCLC. Although few previous studies have reported on the cost impact of lobectomy and its associated complications, ${ }^{7,8}$ in our study we used 90-day direct costs taken from administrative hospital data, rather than extrapolated from national datasets. The incidence of complications observed in this study are similar to those reported in the literature, with some differences. ${ }^{9}$ Some of these differences can be attributed to reporting complications within a 30-day interval rather than 90 days, and to differing definitions of major and minor complications. Our institution is a major referral center, and patients readmitted to outside emergency departments for postoperative complications were not captured in our database, which also might have affected the complication rates observed in this study. We implemented a 90-day interval to capture readmissions that were surrogates of delayed complications related to the primary procedure. We were careful to include a comprehensive list of both major and minor complications tabulated from clinical data rather than administrative data, including pulmonary, cardiovascular, neurologic, gastrointestinal, renal, and infectious complications.

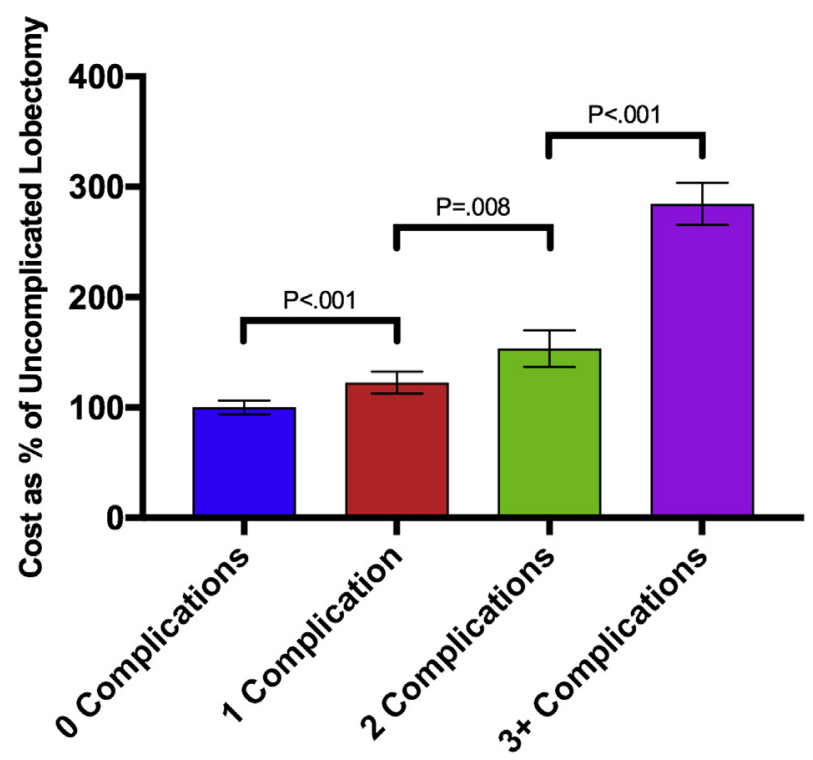

FIGURE 2. Additive impact of increasing complications on mean relative overall cost. Bars reflect means with $95 \%$ confidence intervals (CIs). Relative cost compared to uncomplicated lobectomy: 1 complication, $22.6 \%$ (95\% CI, 12.6\%-32.5\%; $P<.001) ; 2$ complications, $53.4 \%$ (95\% CI, 37.0\%-69.8\%; $P<.001) ; \geq 3$ complications, $184.6 \%(95 \%$ CI, $165.6 \%-203.6 \% ; P<.001)$.

This study identifies several complications associated with significantly increased 90-day direct hospital costs, some due to a high per-event cost impact and others due to a high complication frequency. For example, major pulmonary complications affected only $2.9 \%$ of all patients, but increased the cost of lobectomy by a mean of $111 \%$ above baseline. By contrast, atrial arrhythmia only increased the relative cost of lobectomy by $25 \%$ (partly by increasing hospital LOS), but occurred in $13 \%$ of all patients. Strategies to decrease health care costs while improving patient health outcomes may reasonably target both types of complications.

Enhanced recovery pathways (ERPs) are gained popularity in multiple surgical disciplines to protocolize postoperative care and shorten LOS, but evidence is lacking for their use following pulmonary lobectomy. Madani and colleagues ${ }^{10}$ reported on the impact of an ERP on LOS and complication rates following open lobectomy. Compared with standard postoperative care, the ERP conferred a shorter median LOS (6 vs 7 days; $P<.01$ ) and lower 30 -day complication rate $(37 \%$ vs $50 \%$; $P=.03) .{ }^{10}$ When analyzed in the context of minimally invasive lobectomy, the benefit of ERPs is less compelling, however. Brunelli and colleagues ${ }^{11}$ compared 365 patients receiving standard care and 235 patients receiving ERPs undergoing VATS lobectomy between 2014 and 2017, and found no difference in LOS, cardiopulmonary complication rates, 30- and 90-day mortality, or 30- and 90-day readmission rates between the 2 groups. The authors 


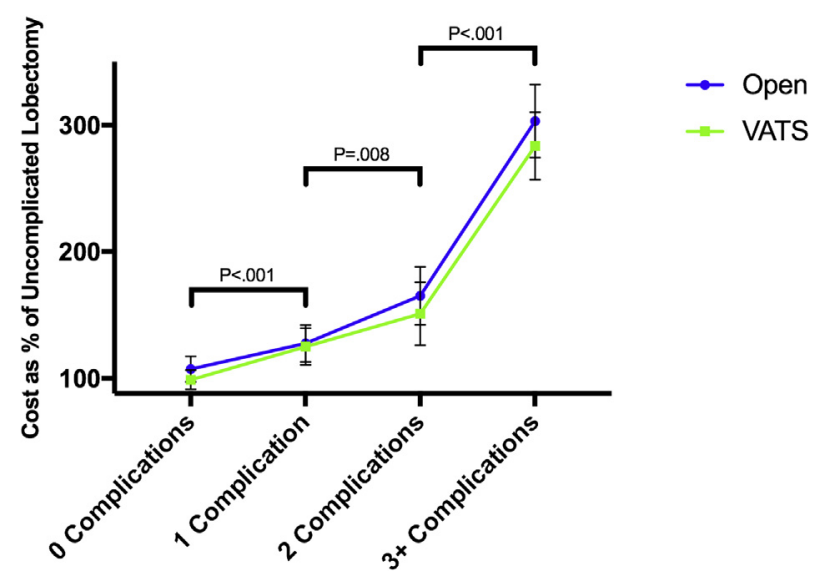

FIGURE 3. Additive impact of increasing complications on mean relative overall cost, separated by surgical approach. Data points reflect means with $95 \%$ confidence intervals. Controlling for number of complications, no significant difference was found in cost between open thoracotomy and video-assisted thoracoscopic surgery (VATS) lobectomy, except at the level of 0 complications, which may be attributable to decreased length of stay in the VATS group.

concluded that "[ERP] elements may be insufficiently different than previous standards of perioperative care to confer detectable benefits in our settings." ${ }^{11}$ Similarly, a recent systematic review and meta-analysis of ERPs in elective lung resections concluded that "some studies suggest [ERPs] may reduce LOS and hospital costs, but they should be interpreted in light of several methodologic limitations." 12 ERPs might not adequately address the specific complications and risk factors most associated with increased LOS and hospital costs, hence their lack of proven efficacy in improving outcomes following lobectomy. Nevertheless, interventions specifically aimed at addressing relevant complications have shown varying degrees of benefit in reducing morbidity after lobectomy.

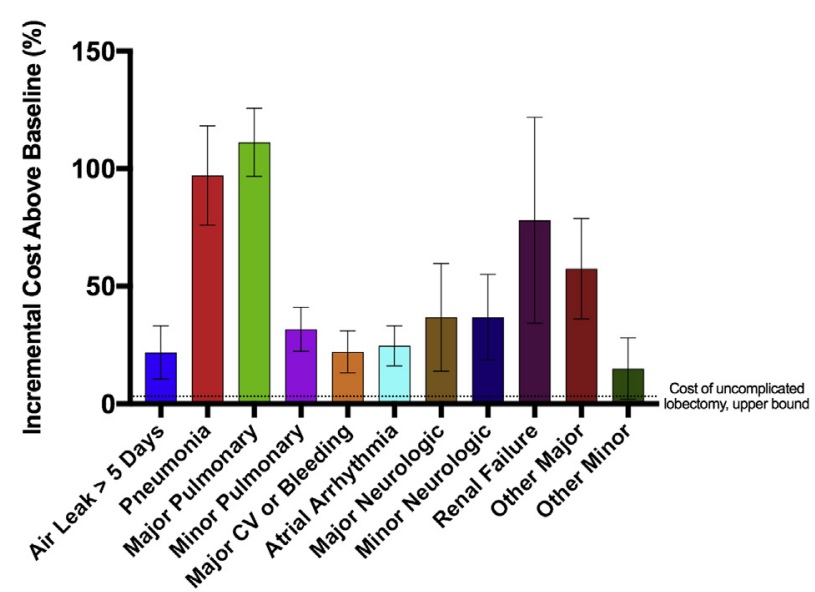

FIGURE 4. Relative incremental cost of specific complications. Bars reflect means with $95 \%$ confidence intervals. $C V$, Cardiovascular.
In a randomized controlled trial comparing preoperative amiodarone to no prophylaxis, Tisdale and colleagues ${ }^{13}$ showed that amiodarone significantly reduced the incidence of atrial fibrillation after pulmonary resection $(13.8 \%$ vs $32.3 \% ; P=.02)$ and also reduced intensive care unit LOS. Unfortunately, there was no difference in hospital LOS and no corresponding decrease in the cost of hospitalization. Of interest, the risk of acute lung injury with short-term use of amiodarone after pulmonary resection (often a reason to avoid its use) has not been substantiated in more recent studies, ${ }^{14}$ but caution remains in patients with pneumonectomy.

The PRESAGE (Prevention of Atrial Fibrillation in High-Risk Patients Undergoing Lung Cancer Surgery) trial randomized 320 patients with elevated N-terminal pro-brain natriuretic peptide to prophylactic treatment with metoprolol or losartan, initiated soon after lung cancer surgery. ${ }^{15}$ The incidence of postoperative atrial fibrillation was significantly lower in the metoprolol and losartan groups compared with the control group $(6 \%, 12 \%$, and $40 \%$, respectively; relative risk, $0.19[P<.001]$ in the metoprolol group vs $0.29[P<.001]$ in the losartan group). Notably, the lower incidence of atrial arrhythmia was associated with a shorter postoperative LOS and a lower incidence of postoperative adverse events.

At least 1 major complication was observed in $17 \%$ of patients who underwent lobectomy. Strategies to curb postoperative complications that should be considered by surgeons and suggested best practices are listed in Table 3. Some of these interventions can be implemented before the operation, such as antiarrhythmia loading in high-risk patients and placement of paravertebral blocks in lieu of epidural catheters. Of note, paravertebral blocks were found to be as effective as thoracic epidurals in a randomized trial assessing postoperative analgesia in VATS lobectomy. ${ }^{16}$ Although a Food and Drug Administration-approved biodegradable lung sealant has been shown to significantly reduce air leaks and LOS in a randomized study, ${ }^{17}$ techniques to reduce postoperative air leak include fissureless dissection when possible, mobilization of all intrapleural adhesions, and mitigation of air leaks intraoperatively. Some accepted interventions to reduce LOS in the management of prolonged postoperative air leak (defined as $>5$ days) include chemical pleurodesis, ${ }^{18}$ autologous blood patches, ${ }^{19}$ and one-way chest tube valves. ${ }^{20}$

To our surprise, the relative cost of complications did not depend on whether patients underwent open or VATS lobectomy (Figure 3); however, patients undergoing VATS lobectomy were overall less likely to experience any complications, even after controlling for performance status and other risk factors (odds ratio, 0.48 ; 95\% CI, 0.30-0.77; $P=.002$ ). Although the mean overall 90-day cost of care was higher in open lobectomy group, this effect was 
TABLE 3. Suggested best practices to reduce complications after lobectomy for NSCLC

\begin{tabular}{ll}
\hline Postoperative complication & \multicolumn{1}{c}{ Suggested best practices } \\
\hline Atrial arrhythmia & - Selected use of prophylactic perioperative amiodarone in patients deemed high risk*13 \\
& - Prophylactic treatment with metoprolol or losartan, initiated soon after lung cancer surgery \\
& in patients with high NT-proBNP levels ${ }^{15}$ \\
Prolonged air leak & - Attempt fissureless dissection when possible \\
& - Mobilization of all intrapleural adhesions \\
& - Routinely check for air leaks at completion of anatomic resection \\
& - Direct suture of large air leaks \\
& - Consider chemical pleurodesis, ${ }^{18}$ autologous blood patch, ${ }^{19}$ or Heimlich valve ${ }^{20}$ in \\
& postoperative air leaks $\geq 5$ d to reduce length of stay \\
Pneumonia, respiratory failure & Minimally invasive lobectomy when possible \\
& - Early ambulation to prevent atelectasis and promote clearance of secretions, head of bed elevation, \\
& incentive spirometry, oral hygiene with chlorhexidine ${ }^{28}$
\end{tabular}

NT-proBNP, $\mathrm{N}$ terminal pro-brain natriuretic peptide. *Age, history of previous heart disease, and preoperative supraventricular arrhythmia in patients undergoing major thoracic operations. ${ }^{26,27}$

mediated by the increased complication rate associated with the open approach compared with VATS, with no significant difference in the per-event cost of complications between the open lobectomy and VATS groups. There was similarly no difference in overall 90-day costs between the open and VATS approaches after controlling for complication rate (Figure 3), except at the level of zero complications. This difference may be explained by the shorter median LOS associated with VATS lobectomy compared with thoracotomy in patients without complications (4 days [IQR, 3-5] vs 5 days $[\mathrm{IQR}, 4-6] ; P=.002$ ). Furthermore, in our univariate linear model, the interaction of surgical approach and total number of complications was not significant, suggesting the estimated cost impact of complications was not affected by surgical approach.

Although no previous prospective randomized controlled trials have compared VATS and open anatomic lung resection for NSCLC, multiple retrospective analyses have demonstrated the superiority of VATS resection in reducing postoperative morbidity. ${ }^{21-23}$ In a review of $>11,000$ patients derived from the Society of Thoracic Surgeons' General Thoracic Surgery database, VATS lobectomy was associated with a significantly lower rate of pulmonary complications compared with thoracotomy $(18 \%$ vs $21 \% ; P=.017) .{ }^{24}$ Accordingly, increased use of a VATS approach may be expected to decrease overall 90-day direct costs vis-à-vis a reduction in complication incidence.

Our study has several limitations. Although we initially identified nearly 1000 patients who underwent lobectomy for early-stage NSCLC in the time period under study, financial data were available for only approximately one half of this cohort. In addition, our study is a major referral center, and patients suffering postdischarge complications may have been treated at a local hospital rather than readmitted to our institution. These complications would be missing from our database. This would mostly affect our observed complication rates, but because all complications captured in our database were treated at our institution, we believe that our cost data are accurate for the complications that we did capture, and thus the relative incremental cost of those complications should be reasonably accurate. Although this study was underpowered to identify the cost of several complications at a significance level of $\alpha=0.05$ due to their low event rate (eg, major infections and minor infections), we show here that all complications do not equally contribute to the cost of care following lobectomy.

We believe that the present study can contribute to the development of future best practice models of lobectomy for NSCLC while addressing modifiable complications. The cost analysis of complications is analogous to the work of the Virginia Cardiac Surgery Quality Initiative, in which 17 state-wide centers reported on outcomes, hospital resource utilization, and costs in patients undergoing coronary artery bypass grafting with or without aortic valve replacement. ${ }^{25}$ Through the process of reporting the cost impact of complications and outcomes in cardiac surgery, the authors spawned several initiatives aimed at improving overall surgical quality within the Commonwealth of Virginia. As a consequence, protocol-driven approaches were implemented to address the high-cost complications, a goal that we strive to translate to lobectomy for NSCLC.

Efforts to reduce health care costs while improving outcomes should focus on the leverage points identified in this study - that is, complications for which an incidence reduction is expected to yield the greatest proportional cost impact reduction. Further analysis is needed to identify the cost of low event rate complications using larger cohorts. 


\section{Conflict of Interest Statement}

Authors have nothing to disclose with regard to commercial support.

\section{References}

1. Muñoz E, Muñoz W III, Wise L. National and surgical health care expenditures, 2005-2025. Ann Surg. 2010;251:195-200.

2. Chassin MR, Galvin RW. The urgent need to improve health care quality. Institute of Medicine National Roundtable on Health Care Quality. JAMA. 1998;280:1000-5

3. Birkmeyer JD, Gust C, Dimick JB, Birkmeyer NJ, Skinner JS. Hospital quality and the cost of inpatient surgery in the United States. Ann Surg. 2012;255:1-5.

4. Dimick JB, Chen SL, Taheri PA, Henderson WG, Khuri SF, Campbell DA Jr. Hospital costs associated with surgical complications: a report from the private-sector National Surgical Quality Improvement Program. J Am Coll Surg. 2004; 199:531-7.

5. Dimick JB, Pronovost PJ, Cowan JA, Lipsett PA. Complications and costs after high-risk surgery: where should we focus quality improvement initiatives? J Am Coll Surg. 2003;196:671-8.

6. Handy JR Jr, Denniston K, Grunkemeier GL, Wu YX. What is the inpatient cost of hospital complications or death after lobectomy or pneumonectomy? Ann Thorac Surg. 2011;91:234-8.

7. Brunelli A, Drosos P, Ismail H, Pompili C, Bassi V. Factors associated with postoperative costs following anatomic lung resections without major complications. Eur J Cardiothorac Surg. 2017;51:230-5.

8. Jawitz OK, Boffa DJ, Detterbeck FC, Wang Z, Kim AW. Estimating the annual incremental cost of several complications following pulmonary lobectomy. Semin Thorac Cardiovasc Surg. 2016;28:531-40.

9. Falcoz PE, Puyraveau M, Thomas PA, Decaluwe H, Hürtgen M, Petersen RH, et al. Video-assisted thoracoscopic surgery versus open lobectomy for primary non-small-cell lung cancer: a propensity-matched analysis of outcome from the European Society of Thoracic Surgeon database. Eur J Cardiothorac Surg. 2016;49:602-9.

10. Madani A, Fiore JF Jr, Wang Y, Bejjani J, Sivakumaran L, Mata J, et al. An enhanced recovery pathway reduces duration of stay and complications after open pulmonary lobectomy. Surgery. 2015;158:899-908; discussion 908-10.

11. Brunelli A, Thomas C, Dinesh P, Lumb A. Enhanced recovery pathway versus standard care in patients undergoing video-assisted thoracoscopic lobectomy. J Thorac Cardiovasc Surg. 2017;154:2084-90.

12. Fiore JF Jr, Bejjani J, Conrad K, Niculiseanu P, Landry T, Lee L, et al. Systematic review of the influence of enhanced recovery pathways in elective lung resection. J Thorac Cardiovasc Surg. 2016;151:708-15.e6.

13. Tisdale JE, Wroblewski HA, Wall DS, Rieger KM, Hammoud ZT, Young JV, et al. A randomized trial evaluating amiodarone for prevention of atrial fibrillation after pulmonary resection. Ann Thorac Surg. 2009;88:886-93; discussion 894-5.

14. Riber LP, Larsen TB, Christensen TD. Postoperative atrial fibrillation prophylaxis after lung surgery: systematic review and meta-analysis. Ann Thorac Surg. 2014;98:1989-97.

15. Cardinale D, Sandri MT, Colombo A, Salvatici M, Tedeschi I, Bacchiani G, et al. Prevention of atrial fibrillation in high-risk patients undergoing lung cancer surgery: the PRESAGE trial. Ann Surg. 2016;264:244-51.
16. Kosiński S, Fryźlewicz E, Wiłkojé M, Ćmiel A, Zieliński M. Comparison of continuous epidural block and continuous paravertebral block in postoperative analgaesia after video-assisted thoracoscopic surgery lobectomy: a randomized, non-inferiority trial. Anaesthesiol Intensive Ther. 2016;48: 280-7.

17. Allen MS, Wood DE, Hawkinson RW, Harpole DH, McKenna RJ, Walsh GL, et al. Prospective randomized study evaluating a biodegradable polymeric sealant for sealing intraoperative air leaks that occur during pulmonary resection. Ann Thorac Surg. 2004;77:1792-801.

18. Liberman M, Muzikansky A, Wright CD, Wain JC, Donahue DM, Allan JS, et al Incidence and risk factors of persistent air leak after major pulmonary resection and use of chemical pleurodesis. Ann Thorac Surg. 2010;89:891-7; discussion 897-8.

19. Droghetti A, Schiavini A, Muriana P, Comel A, De Donno G, Beccaria M, et al Autologous blood patch in persistent air leaks after pulmonary resection. J Thorac Cardiovasc Surg. 2006;132:556-9.

20. McKenna RJ Jr, Fischel RJ, Brenner M, Gelb AF. Use of the Heimlich valve to shorten hospital stay after lung reduction surgery for emphysema. Ann Thorac Surg. 1996;61:1115-7.

21. Swanson SJ, Meyers BF, Gunnarsson CL, Moore M, Howington JA, Maddaus MA, et al. Video-assisted thoracoscopic lobectomy is less costly and morbid than open lobectomy: a retrospective multi-institutional database analysis. Ann Thorac Surg. 2012;93:1027-32.

22. Farjah F, Backhus LM, Varghese TK, Mulligan MS, Cheng AM, AlfonsoCristancho R, et al. Ninety-day costs of video-assisted thoracic surgery versus open lobectomy for lung cancer. Ann Thorac Surg. 2014;98:191-6.

23. Howington JA, Gunnarsson CL, Maddaus MA, McKenna RJ, Meyers BF, Miller D, et al. In-hospital clinical and economic consequences of pulmonary wedge resections for cancer using video-assisted thoracoscopic techniques vs traditional open resections: a retrospective database analysis. Chest. 2012;141: 429-35.

24. Boffa DJ, Dhamija A, Kosinski AS, Kim AW, Detterbeck FC, Mitchell JD, et al Fewer complications result from a video-assisted approach to anatomic resection of clinical stage I lung cancer. J Thorac Cardiovasc Surg. 2014;148: 637-43.

25. LaPar DJ, Crosby IK, Rich JB, Fonner E Jr, Kron IL, Ailawadi G, et al; Investigators for Virginia Cardiac Surgery Quality Initiative. A contemporary cost analysis of postoperative morbidity after coronary artery bypass grafting with and without concomitant aortic valve replacement to improve patient quality and cost-effective care. Ann Thorac Surg. 2013;96:1621-7.

26. Elrakhawy HM, Alassal MA, Elsadeck N, Shaalan A, Ezeldin TH, Shalabi A Predictive factors of supraventricular arrhythmias after noncardiac thoracic surgery: a multicenter study. Heart Surg Forum. 2014;17:E308-12.

27. Cardinale D, Colombo A, Sandri MT, Lamantia G, Colombo N, Civelli M, et al Increased perioperative N-terminal pro-B-type natriuretic peptide levels predic atrial fibrillation after thoracic surgery for lung cancer. Circulation. 2007;115 1339-44.

28. Kazaure HS, Martin M, Yoon JK, Wren SM. Long-term results of a postoperative pneumonia prevention program for the inpatient surgical ward. JAMA Surg. 2014;149:914-8

Key Words: cost, surgical outcomes, lung cancer, lobectomy 